\title{
Connecting to Create: Expertise in Musical Improvisation Is Associated with Increased Functional Connectivity between Premotor and Prefrontal Areas
}

\author{
Ana Luísa Pinho, ${ }^{1,3}$ Örjan de Manzano, ${ }^{1}$ Peter Fransson, ${ }^{2}$ Helene Eriksson, ${ }^{1}$ and Fredrik Ullén ${ }^{1}$ \\ ${ }^{1}$ Department of Neuroscience and ${ }^{2}$ Department of Clinical Neuroscience, Karolinska Institutet, SE-171 77 Stockholm, Sweden; and ${ }^{3} \mathrm{PhD}$ Programme in \\ Experimental Biology and Biomedicine, Center for Neuroscience and Cell Biology, University of Coimbra, Portugal
}

\begin{abstract}
Musicians have been used extensively to study neural correlates of long-term practice, but no studies have investigated the specific effects of training musical creativity. Here, we used human functional MRI to measure brain activity during improvisation in a sample of 39 professional pianists with varying backgrounds in classical and jazz piano playing. We found total hours of improvisation experience to be negatively associated with activity in frontoparietal executive cortical areas. In contrast, improvisation training was positively associated with functional connectivity of the bilateral dorsolateral prefrontal cortices, dorsal premotor cortices, and presupplementary areas. The effects were significant when controlling for hours of classical piano practice and age. These results indicate that even neural mechanisms involved in creative behaviors, which require a flexible online generation of novel and meaningful output, can be automated by training. Second, improvisational musical training can influence functional brain properties at a network level. We show that the greater functional connectivity seen in experienced improvisers may reflect a more efficient exchange of information within associative networks of importance for musical creativity.
\end{abstract}

Key words: Creativity; expertise; fMRI; improvisation; music; plasticity

\section{Introduction}

Creative products are by definition both novel and meaningful. Correspondingly, creative cognition is commonly assumed to involve the free generation of possible solutions as well as selection among the produced alternatives (Campbell, 1960). The neuropsychology of creativity has been studied using tasks ranging from pseudorandom generation of simple responses to the production of musical or verbal materials (Frith, 2000; NathanielJames and Frith, 2002; Lau et al., 2004). Interestingly, pseudorandom and musical generation appear to rely on a common set of regions, which includes the dorsolateral prefrontal cortex (DLPFC), the anterior cingulate cortex, the presupplementary motor area (preSMA), and the inferior frontal gyrus (IFG; de Manzano and Ullén, 2012b). These regions presumably fulfill several cognitive functions during creative thinking, including attention to action (Lau et al., 2004), response generation (Lau et

Received Nov. 11, 2013; revised Feb. 22, 2014; accepted March 20, 2014.

Author contributions: A.L.P., O.d.M., and F.U. designed research; A.L.P. and Ö.d.M. performed research; A.L.P., 0̈.d.M., P.F., H.E., and F.U. analyzed data; F.U. wrote the paper.

This work was supported by the Foundation for Science and Technology (Grabt SFRH/BD/33895/2009), the Swedish Research Council (Grant 521-2010-3195), and the Sven and Dagmar Salén Foundation. We thank Jonathan Berrebi for technical support and comments on the manuscript, László Harmat and Diana Muessgens for assistance during experiments, and Miguel Castelo-Branco for valuable discussions.

The authors declare no competing financial interests.

This article is freely available online through the J Neurosci Author Open Choice option.

Correspondence should be addressed to Fredrik Ullen, Department of Neuroscience, Retzius v. 8, Karolinska Institutet, SE-171 77 Stockholm, Sweden. E-mail: Fredrik.Ullen@ki.se.

DOI:10.1523/JNEUROSCI.4769-13.2014

Copyright $\odot 2014$ the authors $\quad 0270-6474 / 14 / 346156-08 \$ 15.00 / 0$ al., 2004; Bengtsson et al., 2007), action planning and monitoring (Nathaniel-James and Frith, 2002), and inhibition of repetitive responses (Frith, 2000). Studies do not suggest a simple mapping in which different cognitive processes are subserved by distinct cortical subregions. Rather, most involved brain regions appear to interact in several such processes, indicating that creative thinking relies on distributed networks (de Manzano and Ullén, 2012b).

Musical training can have dramatic effects on the brain (Schneider et al., 2002; Bengtsson et al., 2005; Kleber et al., 2010; Pantev and Herholz, 2011). Many correlates of training presumably reflect task automation and the acquisition of specific expertise-related skills. Although several studies have investigated the neural effects of musical training, there is, to our knowledge, no study on the specific consequences of training musical creativity (i.e., improvisation). A key difference between creative and reproductive performance is that the former to a higher degree appears to rely on the executive frontal circuits (Bengtsson et al., 2007; de Manzano and Ullén, 2012b). Therefore, an interesting question is to what extent, if at all, neural circuits involved in creativity can be optimized by systematic training.

Here, we investigated this issue in a sample of 39 pianists with a wide range of experience in both classical piano playing and improvisation. Brain activity was measured with fMRI while the pianists performed brief keyboard improvisations. Total hours of classical piano playing and improvisation were assessed using a self-report questionnaire. We addressed two questions. First, to test whether creative performance is subject to similar training effects as reproductive performance, we investigated whether 
A

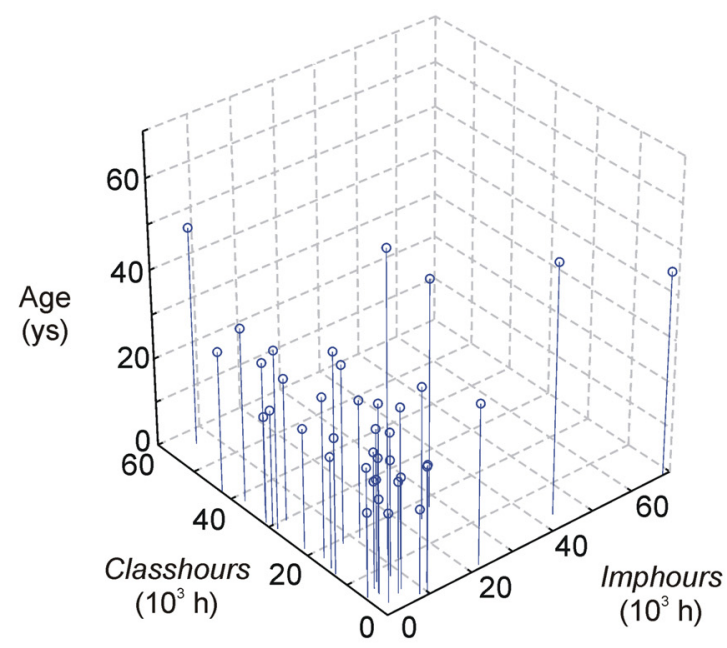

B

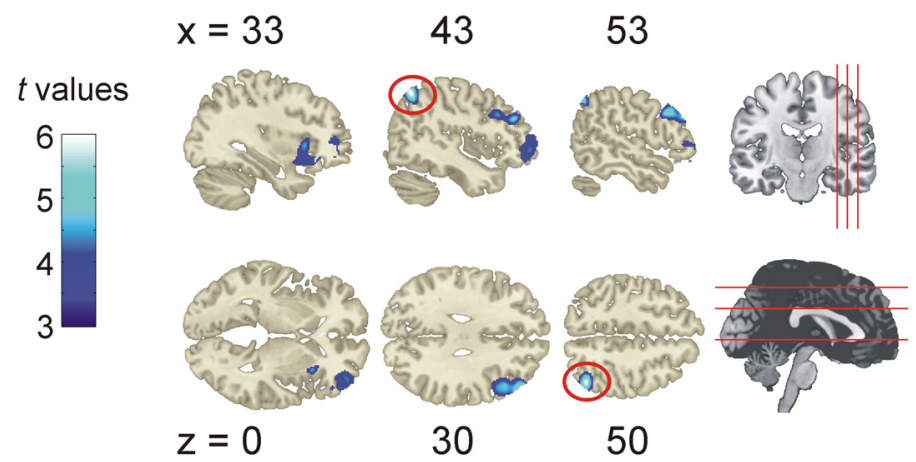

C

\section{$\%$ BOLD signal change in $A G$}

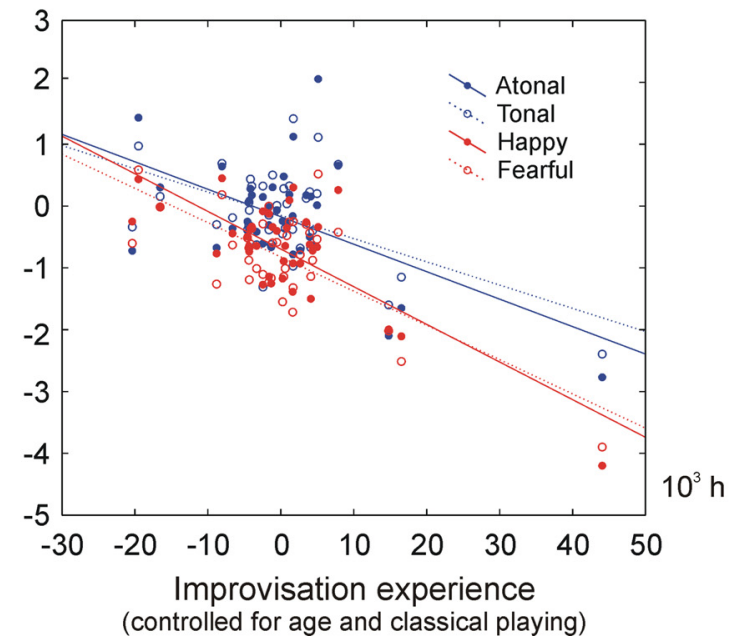

Figure 1. Piano experience and brain activity during improvisation. $\boldsymbol{A}, 3 \mathrm{D}$ scatter plot showing the age, improvisational piano experience (Imphours), and classical piano experience (Classhours) of each participant. $\boldsymbol{B}$, Negative associations between brain activity and improvisational training. The clusters represent regions for which there was a significant between-participant negative association between brain activity during improvisation and number of hours of improvisation experience. C, Brain activity and improvisation experience for individual conditions. The plot shows, for all four individual conditions (tonal, atonal, happy, and fearful), the semipartial correlation between brain activity (BOLD percentage signal change in Improvisation - Rest) in the right angular gyrus (red circle in $\boldsymbol{B}$ ) and Imphours after partialling out the effects of age and Classhours on the latter variable.

frontoparietal brain activity during improvisation was negatively associated with experience in improvisation. Second, creativity requires an integration of different types of information, which can result in the discovery of new and interesting combinations of familiar elements (Campbell, 1960). Given this, it appears reasonable to hypothesize that experienced creators have more extensive task-specific functional connectivity between the distributed neural circuits that are involved in creative performance. To test this, we investigated whether experience in improvisation was correlated with functional connectivity during improvisation. This analysis was based on six seed brain regions known to be involved in free generation of behavior: the right and left preSMA, dorsal premotor cortex (PMD), and DLPFC.

\section{Materials and Methods}

\section{Participants}

Thirty-nine pianists ( 15 female) participated in the study, ranging in age between 19 and 67 years $($ mean $=32.4, \mathrm{SD}=11.0)$. Handedness was determined with the Swedish version of the Edinburgh Handedness Inventory (Oldfield, 1971). All participants, who were recruited by poster advertisements, were right-handed, healthy, and had no history of neurological or psychiatric illness. The participants had varied experience in classical and/or jazz piano playing; all had piano as first instrument and, except for one (a self-taught jazz piano player), all pianists also had a university degree in piano performance or were students at the Royal College of Music in Stockholm, the Guildhall School of Music and Drama in London, or the Nordic Masters in Jazz program (Nomazz) at the Norwegian University of Science and Technology in Trondheim. All participants were active as performers. The experimental procedures were undertaken with the informed written consent of each participant according to Declaration of Helsinki and were approved by the Regional Ethical Review Board in Stockholm (Dnr 2011/637-32 and Dnr 2011/168232). Participants were reimbursed with 600 Swedish Krona.

\section{Materials}

Piano experience questionnaire. The participants were asked to fill out a brief questionnaire about how much they had played the piano throughout their lifetime. Experience was estimated as the mean intensity of playing (hours/week) in three age periods: (1) age $<11$ years, (2) age 12-17 years, and (3) age 18 years to present. Separate estimates were given for improvisation and total piano playing. From these data, estimates were calculated of the total number of hours spent improvising (Imphours), total experience (Totalhours), and experience in classical (nonimprovisatory) piano playing (Classhours $=$ Totalhours - Imphours). Only Imphours and Classhours, that is, total training of improvisation and classical piano playing, were used in the data analysis. To evaluate the test-retest reliability of the practicing data, we performed a second administration of the questionnaire on total piano playing throughout lifetime as part of 
an independent Internet-based data collection. Thirty of our original 39 participants chose to participate in this second data collection, which took place $10-18$ months $($ mean $=13.8, \mathrm{SD}=2.3$ ) after the first data collection.

Piano keyboard, auditory feedback, and musical recordings. The participants performed on a custom designed MR-compatible fiber optic piano keyboard (LUMItouch) of one octave (12 authentic keys ranging from $\mathrm{F}$ to E) during scanning. The keyboard was connected to an opticalelectrical converter placed in the control room. The converter was connected to a MIDI-keyboard (Midistart-2 Pro Keys; Miditech) generating an MIDI signal, which was subsequently sent to a MIDI patchbay/processor (MX-8; Digital Music) producing two different MIDI outputs. The first output was relayed to a sound module (SD-50; Roland), which synthesized the piano sound (GM2; European Pf). This device was connected to the audio system of the MR scanner to provide audio feedback (keys F2-E3) to the participants. The second output was relayed to an external sound card, which was in turn connected to a PC recording the musical samples with a music production software (Cubase 5; Steinberg).

Visual stimuli. The visual stimuli consisted of a set of images providing instructions on which conditions to perform. They were presented at the beginning of each experimental trial. The display was controlled by a custom made E-Prime script (E-Prime 2.0 Professional; Psychological Software Tools). The instructions were presented on MR-compatible OLED display goggles (Nordic Neuro Lab's visual system) mounted on the head coil.

MRI scanner. The fMRI data were acquired using a 3T scanner (3T Discovery MR750; GE) with a 32-channel coil (MR Instruments) at the MR center of the Karolinska Hospital.

\section{Experimental procedure}

Upon arrival at the MR center, the participants were first rebriefed about the purpose of the study and safety procedures, after which they completed a screening form regarding general health and history of disease, as well as the piano experience questionnaire and the informed consent. Finally, they were given detailed instructions on how to perform the experimental conditions in a training session outside of the scanner room. During practice, the participants were seated in a chair with a laptop on the desk in front of them for the presentation of the stimuli and the piano keyboard was positioned on their right side on the desk. The participants were instructed that they should play simple musical improvisations, under different constraints, with the right hand on the keyboard. They were informed that they were allowed to improvise freely as long as they followed the constraints of the condition and that they should continue to play throughout the duration of the trial. In addition, they were instructed not look to the piano keys or their right hand while playing, as would be the case inside the scanner. They then completed one training session that was similar in procedure, visual stimuli, and audio feedback to the real experiment. In total, the training lasted for $\sim 30 \mathrm{~min}$. No difficulties with executing the paradigm were observed by the experimenters nor reported by the participants. The participants were scanned in the supine position with the keyboard placed on their lap. The right arm was supported by a sponge pad to avoid fatigue and to minimize arm movements. Ear plugs and headphones were used to reduce scanner noise and to allow auditory feedback from the piano and verbal communication with the experimenters. The volume of the auditory feedback and dioptric settings in the goggles were adjusted for each participant.

\section{Experimental design}

The experiment was performed in six sessions. Each session consisted of 16 trials arranged in a block design. Each trial was composed of four consecutive parts: (1) instruction (3.5 s), (2) performance (15 s), (3) a distractor task (11 s), and (4) rest (6 s). Therefore, one trial lasted for $35.5 \mathrm{~s}$.

During the instruction session, a visual instruction slide was presented. Four different improvisation conditions were used with different instruction slides. The four conditions differed in the type of constraints the participant should adhere to when improvising. In two conditions (tonal and atonal), a structural constraint was used. The participant was
Table 1. Associations between brain activity during improvisation, improvisation experience, and age

\begin{tabular}{|c|c|c|c|c|c|c|c|c|}
\hline \multirow[b]{2}{*}{ Region } & \multirow[b]{2}{*}{ Side } & \multicolumn{3}{|c|}{ Coordinates $^{a}$} & \multicolumn{2}{|c|}{ Voxel level $^{b}$} & \multicolumn{2}{|c|}{ Cluster level $^{c}$} \\
\hline & & $x$ & $y$ & $z$ & $t$ & $p$ & $p$ & $k_{E}$ \\
\hline \multicolumn{9}{|c|}{ Improvisation (negative) } \\
\hline$A G$ & Right & 44 & -61 & 52 & 5.98 & 0.008 & - & - \\
\hline DLPFC & Right & 48 & 38 & 30 & - & - & 0.035 & 1322 \\
\hline Insula & Right & 33 & 18 & 1 & - & - & 0.039 & 1273 \\
\hline IFG & Right & 39 & 50 & -6 & - & - & 0.028 & 1419 \\
\hline \multicolumn{9}{|c|}{ Age (positive) } \\
\hline$A G$ & Right & 48 & -54 & 40 & - & - & 0.035 & 1325 \\
\hline DLPFC & Right & 51 & 33 & 25 & 5.43 & 0.033 & 0.025 & 1459 \\
\hline IFG & Right & 44 & 48 & -17 & 5.38 & 0.037 & 0.004 & 2295 \\
\hline
\end{tabular}

$A G$, angular gyrus; BA, Brodmann's area.

${ }^{a}$ Coordinates in millimeters in the Montreal Neurological Institute space.

${ }^{b}$ Voxel-level familywise error rate, corrected $p=0.05$.

'Cluster-level familywise error rate, corrected extent threshold $k_{E}=1273$ (improvisation) and $k_{E}=1325$ (age).

instructed to improvise using only six different pitches. These were displayed as six whole notes, notated on a single musical staff. In tonal, the pitches were all from the same Western musical scale (major, minor). The pitches thus naturally suggested a tonality for the improvisation. Pitch sets for atonal, in contrast, were randomly generated to fulfill two different criteria: (1) that the pitches were not all part of the same major or minor scale and (2) that there would be at least one interval equal to or larger than a minor third (to avoid chromatic sequences). Therefore, this pitch set did not suggest any particular tonality. The specific pitch sets were unique for each trial of tonal and atonal. For the two other conditions (happy and fearful), the participant was instructed to produce an improvisation with the corresponding emotional character. The instruction slide for these conditions showed a happy or fearful clip art face. The order of trials were randomized with the constraint that the same condition could never appear in more than two consecutive trials and that no more than three consecutive trials would consist of conditions in the same category (structural or emotional). Each condition (tonal, atonal, happy, and fearful) occurred two times in each session. Because correlations between brain activity and improvisation experience were similar in all four improvisation conditions (Fig. 1B), data were pooled across conditions in the present study. The participants had been instructed to commence the performance once the instruction phase was over; that is, when the instruction slide disappeared after $3.5 \mathrm{~s}$.

The performance phase was interrupted by the distractor task. This task was included to interrupt musical cognitive processes from the previous trial and to minimize planning of the next improvisation. The distractor task was constructed as a visual esthetical judgment task. The participant would view a fixation cross for $3 \mathrm{~s}$ and then watch a computer-generated image for $5 \mathrm{~s}$. Then, during the final $3 \mathrm{~s}$ of the task, they were to provide a rating of the aesthetic quality of the image by pressing on the keys on the piano (higher note $=$ higher rating). Results from the analyses of this task will be presented in a separate manuscript.

\section{Data acquisition}

All behavioral (musical) data were recorded in MIDI format and analyzed by a custom-made script in MATLAB 7 (The MathWorks). The fMRI data were collected using a gradient echo pulse, EPI T2*-weighted sequence with BOLD contrasts using the following parameters: $\mathrm{TR}=$ $2.5 \mathrm{~s} ; \mathrm{TE}=30 \mathrm{~ms}$; flip angle $=90^{\circ} ; \mathrm{FOV}=28.8 \mathrm{~cm}$; slice spacing $=0 \mathrm{~mm}$; voxel size $=3 \times 3 \times 3 \mathrm{~mm}^{3}$; data acquisition matrix $=96 \times 96$, interpolated during reconstruction to $128 \times 128$; slice order $=$ interleaved; number of slices $=48$. A total of 228 functional image volumes were acquired per session, giving a total of 1368 image volumes per participant. At the beginning of each session, 10 "dummy" image volumes were scanned, but not saved, to allow for T1-equilibration effects. Subsequently, a 3D fast-spoiled gradient echo T1-weighted anatomical image volume covering the whole brain was acquired: voxel size $=1 \times 1 \times 1$ $\mathrm{mm}^{3}$; axial slice orientation; flip angle $=12^{\circ}$; inversion time $=450 \mathrm{~ms}$; $\mathrm{FOV}=24 \mathrm{~cm}$. 

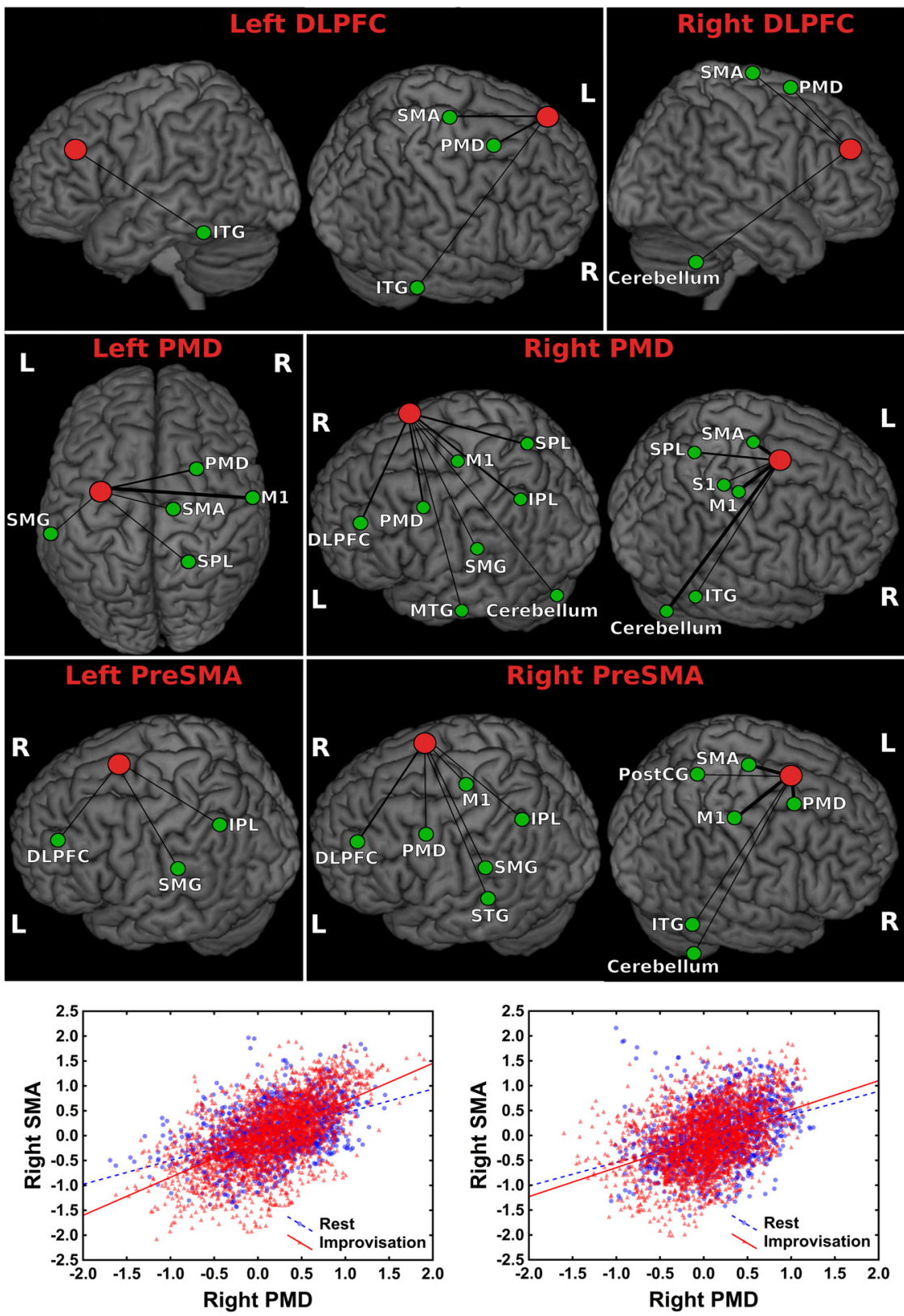

Figure 2. Positive correlations between improvisational training and functional connectivity. Associations between functional connectivity and improvisational training are illustrated schematically for all seed regions. The red circles indicate the location of the seeds. Green circles represent regions for which a significant between-subject correlation was found between improvisational training and the strength of the functional connectivity with the seed. For each involved region, only the cluster with the highest peak activation is illustrated. The thickness of the connecting lines represents the strength of the effect (thin: $t$ value $5-6$; middle: $t$ value $6-7$; thick: $t$ value $>7$ ). The scatterplots show activity (\% BOLD response) in the right SMA as a function of activity in the right PMD during improvisation (red dots) and rest (blue dots) for the participant with the longest (left) and shortest (right) improvisation experience. The increase in functional connectivity during improvisation, compared with rest, is higher for the participant with longer experience, which is illustrated by a greater increase in slope of the ordinary least-squares fitted regression line for improvisation (red) compared with rest (blue).

\section{Data analysis}

Analysis of behavioral data. For the structural conditions, criteria for distinguishing "good" (i.e., when the participant adhered to the structural constraint with a reasonable degree of correctness) from "bad" performances were used. A good performance corresponded to a trial in which the participant used at least five of the presented pitches and used at maximum one wrong pitch. In this way, we removed performances in which the participant held an incorrect representation of the instructions but at the same time allowed for small involuntary slips. The excluded bad performances accounted for $\sim 9 \%$ of the data in the analysis; $2 \%$ of the collected data were removed due to various technical problems, for example, with MIDI recordings.

Three different measures were used to characterize the complexity of the musical samples: 0 -order melodic entropy (considering the distribution of single notes), 1-order melodic entropy (considering the distribution of bigrams of two consecutive pitches), and the LempelZiv complexity measure. Entropy measures were calculated as Shannon entropies as follows:

0-order entropy was calculated as follows:

$$
H=-\sum_{i=1}^{n} p\left(x_{i}\right) \log _{2} p\left(x_{i}\right)
$$

1-order entropy was calculated as follows:

$H=-\sum_{i=1}^{n} p\left(x_{i}\right) \sum_{j=1}^{n} p\left(x_{j} \mid x_{i}\right) \log _{2} p\left(x_{j} \mid x_{i}\right)$

where $H$ is the entropy, $n$ is the number of different elements present in the sequence, and $x_{i}$ is the $i:$ th of these elements. The probabilities $p\left(x_{i}\right)$ and the conditional probabilities $p\left(x_{j} \mid x_{i}\right)$ were estimated from the frequencies of the corresponding elements and bigrams twice, respectively. The Lempel-Ziv complexity is a measure of the number of unique patterns present in a sequence (Doganaksoy and Göloglu, 2006).

Preprocessing of fMRI data. The MRI data were processed and analyzed using the SPM8 software package (Wellcome Department of Imaging Neuroscience, London). For each participant, all fMRI image volumes were realigned to the first image of the first session and resliced using a third-degree B-spline interpolation scheme (Friston et al., 1995). To remove residual variance caused by susceptibility by movement interaction, the fMRI images were afterward unwarped with a fourth-degree B-spline interpolation sampling transformation (Andersson et al., 2001). The T1-weighted anatomical image was then coregistered onto the fMRI unwarped mean image (Ashburner and Friston, 1997). The Brain Extraction Tool was used to remove nonbrain tissue from the anatomical image (Smith, 2002). This image was then segmented to estimate the deformation field for the normalization of all functional and anatomical images (Ashburner and Friston, 2005).

fMRI analyses. The fMRI data were modeled using a general linear model (GLM) using the standard hemodynamic response function (HRF) as implemented in SPM8. At the first level, five regressors of interest were included: (1) instruction, corresponding to the initial instruction phase before the improvisation; (2) improvisation, corresponding to the performance phase of the good performances (see Analysis of behavioral data); (3) bad performances, corresponding to the performance phase of the bad performances; (4) distractor presentation, corresponding to the initial phase (fixation cross and image presentation) of the esthetical judgment task; and (5) distractor rating, corresponding to the final phase of the distractor task in which the participant rated the esthetical quality of the presented image. Rest was modeled implicitly. The high-pass filter was set to $71 \mathrm{~s}$, or $2 \times$ the condition duration. The design matrix weighted each preprocessed im- 
age according to its overall variability to reduce the impact of movement artifacts (Diedrichsen and Shadmehr, 2005). For a few participants, one or two sessions were excluded as a consequence of the inclusion criteria for good performances. For one participant, four sessions were thus used and, for three other participants, five sessions were used. For the remaining 35 participants, all six sessions were used. Contrasts were weighted with the number of included sessions. The contrast images were smoothed using a Gaussian kernel with a full-width-at-half-maximum (FWHM) of $10 \mathrm{~mm}$.

A multiple regression at the second level was then performed, regressing the first-level beta estimates of the Improvisation regressor on age, Imphours, and Classhours. A gray matter mask was estimated during the segmentation process for each participant. The mean of these images was used as an explicit mask in the second-level analysis. The significance of effects was assessed using $t$ statistics to create statistical parametric maps.

A psychophysiological interaction (PPI) analysis was performed to analyze differences in functional connectivity between brain regions between the experimental conditions (Friston et al., 1997). Specifically, we tested the hypothesis that functional connectivity during Improvisation, compared with Rest, would be positively related to Imphours. We identified six regions that have been implied in improvisation in earlier studies (de Manzano and Ullén, 2012a; de Manzano and Ullén, 2012b) and that were confirmed to be active during improvisation in the present experiment, which we used as seed regions: the left and right DLPFC, the left and right dorsal premotor area, and the left and right preSMA. The extent of each seed region was determined as the intersection of the corresponding clusters of activity in the GLM contrast Improvisation - Rest (onesample $t$ test) and the respective region as defined by the Human Motor Area Template (HMAT). The HMAT was created from a meta-analysis of 126 studies and describes the spatial extent of various regions in the motor system (Mayka et al., 2006). Because the HMAT does not include the DLPFC, this seed was defined using the corresponding cluster of activity from the contrast Improvisation-Rest.

For each PPI analysis, the BOLD signal of the seed region was deconvolved with the HRF. Second, the PPI was formed by multiplying this neural activity signal with a block regressor representing the conditions Improvisation and Rest as 1 and -1 , respectively. Third, a GLM analysis was performed with all three regressors in the model: the neural activity, the block regressor representing the two conditions, and the PPI. The high-pass filter was set to $71 \mathrm{~s}$. Each preprocessed image was weighted with its overall variability (more variable images receiving a lower weighting) to reduce the impact of movement artifacts (Diedrichsen and Shadmehr, 2005). The resulting contrast images were smoothed with an isotropic Gaussian kernel of $10 \mathrm{~mm}$ FWHM. Last, a second-level random effect analysis was performed using a multiple regression model in which the contrast images were regressed on participant age, Imphours, and Classhours. The mean of the gray matter masks from all participants was used as an explicit mask during this analysis.

\section{Results}

The participants displayed a wide range of both improvisational and classical piano experience: Imphours varied between 0 and $68,120 \mathrm{~h}($ mean $=9100, \mathrm{SD}=13,014)$; Classhours varied between 0 and $56,212 \mathrm{~h}($ mean $=14,685, \mathrm{SD}=13,062)$. Imphours correlated positively with age $(r=0.43, p=0.006)$ and negatively with Classhours $(r=-0.34, p=0.04)$. Both age and Classhours were therefore included as covariates in all analyses. Test-retest data on the retrospective estimates of total piano experience (Totalhours) was available for 30 of the 39 participants. The retesting was administered 10-18 months after the original test and the testretest correlation was $r=0.76(p<0.0001)$.

We found a significant negative correlation between improvisation experience (Imphours) and brain activity during improvisation (Improvisation - Rest) in a number of cortical regions in the right hemisphere: the DLPFC, the IFG, the anterior insula, and angular gyrus (Fig. 1A, Table 1) The negative association between improvisation experience and brain activity was seen for
Table 2. Brain regions for which there was a positive association between improvisation experience and the functional connectivity during improvisation

\begin{tabular}{|c|c|c|c|c|c|c|c|c|}
\hline \multirow[b]{2}{*}{ Seed } & \multirow[b]{2}{*}{ Side } & \multirow[b]{2}{*}{ Region } & \multirow[b]{2}{*}{ Side } & \multicolumn{3}{|c|}{ Coordinates $^{a}$} & \multirow{2}{*}{$\begin{array}{l}\text { Voxel } \\
\text { level }^{b} \\
t\end{array}$} & \multirow{2}{*}{$\begin{array}{l}\text { Cluster } \\
\text { size } \\
k_{E} \\
\end{array}$} \\
\hline & & & & $x$ & $y$ & $z$ & & \\
\hline \multirow[t]{8}{*}{ DLPFC } & \multirow[t]{3}{*}{ Right } & PMD/SFG & Right & 26 & 11 & 69 & 5.81 & 13 \\
\hline & & Cerebellum & Right & 6 & -49 & -36 & 5.49 & 33 \\
\hline & & SMA/PreCG & Right & 14 & -15 & 78 & 5.25 & 7 \\
\hline & \multirow[t]{5}{*}{ Left } & PMD/SFG & Right & 26 & 11 & 69 & 6.37 & 93 \\
\hline & & SMA/FL & Right & 12 & -16 & 78 & 6.34 & 39 \\
\hline & & ITG & Left & -48 & -49 & -21 & 5.68 & 90 \\
\hline & & ITG & Right & 46 & -34 & -24 & 5.38 & 23 \\
\hline & & ITG & Right & 48 & -49 & -23 & 5.26 & 70 \\
\hline \multirow[t]{29}{*}{ PMD } & \multirow[t]{23}{*}{ Right } & SMA/FL & Right & 10 & -13 & 78 & 9.55 & 186 \\
\hline & & Cerebellum & Right & 54 & -67 & -30 & 7.59 & 1569 \\
\hline & & $\mathrm{M} 1 / \mathrm{CS}^{\mathrm{c}}$ & Right & 51 & -18 & 62 & 7.07 & 301 \\
\hline & & Cerebellum & Right & 6 & -52 & -39 & 7.0 & 272 \\
\hline & & $\mathrm{M} 1 / \mathrm{CS}^{d}$ & Right & 63 & -7 & 40 & 6.73 & 284 \\
\hline & & DLPFC/MFG & Left & -36 & 45 & 36 & 6.54 & 113 \\
\hline & & SPL & Right & 15 & -48 & 78 & 6.4 & 255 \\
\hline & & IPL & Left & -50 & -55 & 54 & 6.31 & 96 \\
\hline & & PMD/PreCG & Left & -52 & 3 & 49 & 6.03 & 53 \\
\hline & & Precuneus/SPL & Left & -6 & -60 & 69 & 6.01 & 42 \\
\hline & & M1/PreCG & Left & -34 & -10 & 70 & 5.93 & 130 \\
\hline & & MTG & Left & -69 & -19 & -20 & 5.75 & 14 \\
\hline & & Cerebellum & Right & 9 & -90 & -38 & 5.72 & 22 \\
\hline & & Cerebellum & Right & 18 & -60 & -18 & 5.65 & 45 \\
\hline & & SMG & Left & -68 & -28 & 31 & 5.63 & 32 \\
\hline & & Cerebellum & Left & -16 & -67 & -21 & 5.52 & 38 \\
\hline & & Cerebellum & Right & 14 & -78 & -42 & 5.51 & 68 \\
\hline & & $\mathrm{M} 1 / \mathrm{CS}$ & Left & -24 & -27 & 75 & 5.46 & 4 \\
\hline & & M1/ParaCL & Left & -14 & -24 & 78 & 5.42 & 5 \\
\hline & & S1/PostCG & Right & 30 & -31 & 45 & 5.41 & 16 \\
\hline & & ITG & Right & 45 & -25 & -20 & 5.4 & 28 \\
\hline & & M1/PreCG & Left & -45 & -15 & 63 & 5.36 & 15 \\
\hline & & ITG & Right & 46 & -19 & -14 & 5.25 & 4 \\
\hline & \multirow[t]{6}{*}{ Left } & $\mathrm{M} 1 / \mathrm{CS}^{d}$ & Right & 63 & -7 & 40 & 7.35 & 127 \\
\hline & & $\mathrm{M} 1 / \mathrm{CS}^{\mathrm{C}}$ & Right & 52 & -15 & 58 & 7.17 & 112 \\
\hline & & PMD/SFG & Right & 30 & 12 & 66 & 6.85 & 88 \\
\hline & & SMG & Left & -68 & -30 & 31 & 5.75 & 50 \\
\hline & & SMA/FL & Right & 10 & -13 & 78 & 5.57 & 13 \\
\hline & & SPL & Right & 21 & -46 & 76 & 5.37 & 11 \\
\hline \multirow[t]{18}{*}{ PreSMA } & \multirow[t]{15}{*}{ Right } & SMA/PreCG & Right & 14 & -15 & 78 & 7.18 & 79 \\
\hline & & PMD/SFG & Right & 26 & 11 & 69 & 7.15 & 126 \\
\hline & & $\mathrm{M} 1 / \mathrm{CS}^{\mathrm{c}}$ & Right & 51 & -18 & 62 & 7.02 & 316 \\
\hline & & $\mathrm{M} 1 / \mathrm{CS}^{d}$ & Right & 63 & -7 & 40 & 6.69 & 288 \\
\hline & & DLPFC/MFG & Left & -36 & 45 & 36 & 6.07 & 71 \\
\hline & & IPL & Left & -46 & -57 & 55 & 5.95 & 53 \\
\hline & & SMG & Left & -68 & -30 & 31 & 5.8 & 89 \\
\hline & & Cerebellum & Right & 54 & -67 & -30 & 5.78 & 52 \\
\hline & & STG & Left & -69 & -37 & 12 & 5.69 & 21 \\
\hline & & Cerebellum & Right & 3 & -49 & -39 & 5.68 & 91 \\
\hline & & ITG & Right & 48 & -28 & -23 & 5.54 & 37 \\
\hline & & PostCG & Right & 20 & -46 & 76 & 5.54 & 19 \\
\hline & & M1/PreCG & Left & -28 & -21 & 73 & 5.4 & 14 \\
\hline & & ITG & Right & 52 & -51 & -18 & 5.35 & 38 \\
\hline & & PMD/PreCG & Left & -52 & 5 & 48 & 5.3 & 4 \\
\hline & \multirow[t]{3}{*}{ Left } & $\mathrm{IPL}$ & Left & -48 & -57 & 54 & 5.75 & 66 \\
\hline & & SMG & Left & -68 & -28 & 31 & 5.72 & 40 \\
\hline & & DLPFC/MFG & Left & -39 & 42 & 34 & 5.39 & 10 \\
\hline
\end{tabular}

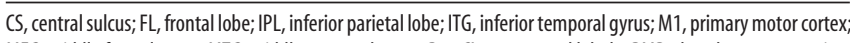
MFG, middle frontal gyrus; MTG, middle temporal gyrus; ParaCL, paracentral lobule; PMD, dorsal premotor region PMV, ventral premotor region; PostCG, postcentral gyrus; PreCG, precentral gyrus; $S 1$, primary somatosensory cortex; SFG, superior frontal gyrus; SMA, supplementary motor area; SPL, superior parietal lobe; STG, superior temporal gyrus; SMG, supramarginal gyrus.

${ }^{a}$ Coordinates in millimeters in the Montreal Neurological Institute space.

${ }^{b}$ Voxel-level familywise error rate, corrected $p=0.05$.

'The cluster extended into the PreCG.

${ }^{d}$ The cluster extended into the PMV/PreCG. 


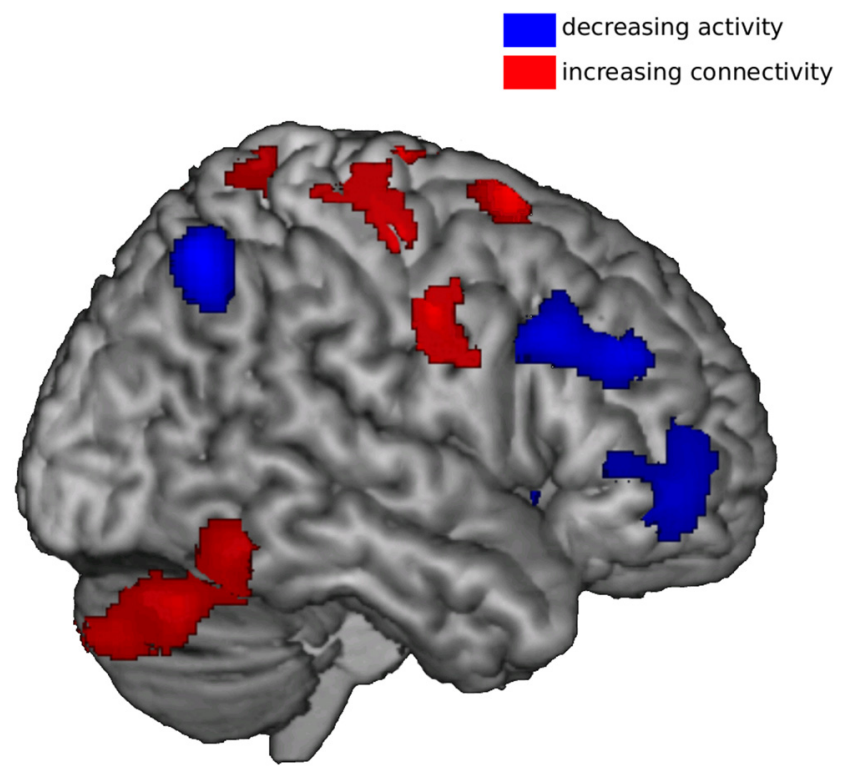

Figure 3. Anatomical relation between training effects on activity and training effects on connectivity. Regions where improvisation experience was related to lower activity (blue) were nonoverlapping with regions showing increased functional connectivity (red). Connectivity effects from all seed regions are displayed.

all individual improvisation conditions, as illustrated for the angular gyrus in Figure $1 B$. In contrast, brain activity during improvisation did not show any significant relation to classical piano training (Classhours). Age was positively related to brain activity in the DLPFC, the right parietal lobe, and the inferior frontal cortex, partly overlapping with the clusters for Imphours.

Second, we found that more experienced improvisers showed higher functional connectivity between prefrontal, premotor, and motor regions of the frontal lobe during improvisation compared with rest. Significant effects were found for each of the six seed regions (Fig. 2, Table 2). Particularly extensive effects were seen when using the right PMD as the seed region (Fig. 2). No negative correlations between Imphours and functional connectivity were found and there were no associations between Classhours and functional connectivity. The brain regions where functional connectivity correlated with Imphours were anatomically nonoverlapping with the regions that showed a lower activity (Fig. 3). Finally, we investigated whether the observed effects of improvisation experience were confounded with the complexity of motor output during improvisations. We found no evidence for this, because Imphours was uncorrelated with all used measures of melodic complexity: Lempel-Ziv string complexity $(r=0.12, p=0.48), 0$-order entropy $(r=0.13, p=0.43), 1$-order entropy $(r=0.12, p=0.47)$, and number of notes of the improvisation $(r=0.28, p=0.09)$.

\section{Discussion}

During improvisation, more trained improvisers displayed an overall lower activity in frontoparietal association areas, as well as greater functional connectivity among prefrontal, premotor, and motor regions of the frontal lobe. The effects were specific to improvisation experience; that is, they were independent of classical piano experience and age, and appeared not to be confounded by differences in the complexity of the musical output between more and less experienced improvisers.

These findings are of general interest in several ways. First, they show that the neural effects of musical training may depend on the type of training in which a musician engages. Earlier studies have typically focused on the effects of conventional, classical musical training. Some of these studies have also provided evidence that neural correlates of musical training differ depending on the instrument trained, with subtle differences between singers and various instrumental players in both the anatomy and functional organization of motor and auditory regions. For example, the relative size of the left and right motor cortices differs between piano and string players (Bangert and Schlaug, 2006), the ultrastructure of the arcuate fasciculus differs between singers and instrumentalists (Halwani et al., 2011), and auditory cortical representations for tones are enhanced in a timbre-specific way for tones from the instrument that the musician has trained (Pantev et al., 2001). The present results provide an example of another type of "specialization of the specialized" (Bangert and Schlaug, 2006), in which the specific effects of improvisation experience presumably relate to cognitive aspects of the training rather than the sensorimotor demands of piano playing.

An important focus of improvisation training is to acquire extensive long-term stores of musical patterns and cognitive strategies that can be used during extemporization (Pressing, 1988). Creativity in improvisation is thus not a matter of free generation of individual notes or sounds, but rather comes into play in the skilled combination, development, and expressive rendering of prelearned musical structures in real time. In an extensive review of improvisation from a cognitive psychological perspective, Pressing (1988) also emphasized the importance of rapid accessibility of musical patterns through the buildup of a rich structure of associations and the integration of simpler structures into larger assemblies, which facilitates complex performances. Consistent with this, we suggest that the greater functional connectivity of the frontal brain regions seen in the most experienced participants may reflect a more efficient integration of representations of musical structures at different levels of abstraction. A higher functional connectivity of the seed regions was observed with premotor regions and parietal and prefrontal association cortex, as well as with primary sensorimotor cortex and the cerebellum, suggesting that the training-related functional reorganizations may affect both cognitive and sensorimotor aspects of improvisation.

The lower overall level of activity in prefrontal and parietal association cortices in highly trained improvisers raises the intriguing question of to what extent a creative behavior such as improvisation can be automated. Sustained attention has been suggested to be critical for creative thinking and for tasks involving the processing of novel information in general (Dietrich, 2004). Both neuroimaging data and experimental interventions with transcranial magnetic stimulation show that prefrontal executive areas are involved in tasks involving free generation (Jahanshahi and Dirnberger, 1999; de Manzano and Ullén, 2012b). Notably, however, de Manzano and Ullén (2012b) found a significantly higher activity in frontoparietal association cortex when contrasting an untrained free generation task (pseudorandom generation) with musical improvisation in musicians. It appears possible, therefore, that long-term training could lead to an automation of the task-specific cognitive processes that are used to generate new and meaningful recombinations of musical structures by expert improvisers. Limb and Braun (2008) reported extensive prefrontal deactivations during free improvisations in a study of six experienced jazz musicians. The regions showing training-related activity decreases were essentially nonoverlapping with the regions showing training-related increases in functional connectivity. The lowered frontoparietal activity, 
indicating automation and reduced top-down cognitive control, was thus accompanied by increased interactions among regions that maintained a higher activity level during performance. In addition, a subjective feeling of automaticity and psychological flow during performance has often been discussed in accounts of performances by improvising musicians (Pressing, 1988). In contrast to the effects of improvisational experience, we found age to be positively associated with frontoparietal activity. This is consistent with many earlier studies finding higher activity in older than in younger subjects in frontoparietal systems during the performance of demanding cognitive (Emery et al., 2008; Salami et al., 2014) and motor (Heuninckx et al., 2008) tasks. Possibly, this tendency for higher activity in executive regions in older participants could represent a compensation for age-related decline in functional capacity (Heuninckx et al., 2008), even though most of the participants in the present study were relatively young adults. The importance of frontoparietal systems for creativity is supported by several studies using EEG to study brain activity during tasks involving creative thinking (Fink and Benedek, 2013). Higher alpha-band power in the EEG activity, in particular at prefrontal and right parietal sites, has been reported during creative (e.g., divergent) compared with less creative tasks (Fink et al., 2009b; Jauk et al., 2012; Fink and Benedek, 2013), and more creative individuals have been found to have higher alpha-band power during creative task performance than less creative individuals (Fink et al., 2009a; Fink and Benedek, 2013). However, one should note that alpha synchronization is not specifically linked to creative thinking, but is also seen in other cognitive tasks such as working memory, and that the EEG literature on creativity shows considerably heterogeneity (Arden et al., 2010).

The present results provide further support for the multifaceted nature of neural correlates of long-term training. Earlier studies have reported that musical training is associated with regional gray matter volume and cortical thickness (Hyde et al., 2009), white matter ultrastructure and connectivity (Bengtsson et al., 2005; Halwani et al., 2011; Steele et al., 2013), and regional differences in the functional organization of brain areas and their level of activity during the performance of musical tasks (Schneider et al., 2002; Kleber et al., 2010; Pantev and Herholz, 2011). The present findings show that musical training also influences functional brain activity at a network level; that is, the patterns of communication between brain regions involved in musical performance. Interestingly, the results indicate that task performance in highly trained individuals compared with novices may involve a lower overall level of regional brain activity that is concomitant with an increase in functional connectivity. Skilled improvisational performance may thus be characterized by both lower demands on executive control and a more efficient interaction within the network of involved brain areas.

The performed improvisations were, necessarily, limited in duration and complexity. However, earlier comparisons between even more reduced musical improvisations (brief isochronous melodies) and pseudorandom response generation on the same keyboard demonstrated significantly higher activity in frontoparietal executive systems during the untrained, pseudorandom task, supporting that even highly simplified forms of musical improvisation tap into musical expertise (de Manzano and Ullén, 2012 b). The fact that brain activity during performance was associated with real-life improvisational experience is in itself further support for the ecological validity of the task. In addition, the effects of improvisational training were consistent across the improvisation conditions. This suggests that the training effects, although specific to improvisational training, were generic in the sense that they were not limited only to certain strategies for improvisation. It appears likely that experienced improvisers not only show a more efficient neural control of improvisation, but also have a higher capacity to perform complex improvisations with a high musical quality. To investigate such differences in improvisational capacity, a design in which participants are allowed to perform more complex and challenging improvisations at the top of their capacity would presumably be needed.

Training data were collected as retrospectively estimated typical training per week in different age periods, which is common practice in expertise studies. Cumulative estimates of total hours of training were for simplicity based on the assumption of 52 training weeks per year. This assumption is obviously somewhat arbitrary and the absolute values of total practicing hours should therefore be viewed with some caution. Musicians typically combine training with concert performances and teaching and a more realistic estimate is presumably lower. Importantly, however, the assumed number of active training weeks per year will of course not influence the associations between training and other variables.

\section{References}

Andersson JL, Hutton C, Ashburner J, Turner R, Friston K (2001) Modeling geometric deformations in EPI time series. Neuroimage 13:903-919. CrossRef Medline

Arden R, Chavez RS, Grazioplene R, Jung RE (2010) Neuroimaging creativity: A psychometric view. Behav Brain Res 214:143-156. CrossRef Medline

Ashburner J, Friston K (1997) Multimodal image coregistration and partitioning-a unified framework. Neuroimage 6:209-217. CrossRef Medline

Ashburner J, Friston KJ (2005) Unified segmentation. Neuroimage 26:839851. CrossRef Medline

Bangert M, Schlaug G (2006) Specialization of the specialized in features of external human brain morphology. Eur J Neurosci 24:1832-1834. CrossRef Medline

Bengtsson SL, Nagy Z, Skare S, Forsman L, Forssberg H, Ullén F (2005) Extensive piano practicing has regionally specific effects on white matter development. Nat Neurosci 8:1148-1150. CrossRef Medline

Bengtsson SL, Csíkszentmihályi M, UIlén F (2007) Cortical regions involved in the generation of musical structures during improvisation in pianists. J Cogn Neurosci 19:1-13. CrossRef Medline

Campbell DT (1960) Blind variation and selective retention in creative thought as in other knowledge processes. Psychol Rev 67:380-400. CrossRef Medline

de Manzano Ö, Ullén F (2012a) Activation and connectivity patterns of the presupplementary and dorsal premotor areas during free improvisation of melodies and rhythms. Neuroimage 63:272-280. CrossRef Medline

de Manzano Ö, Ullén F (2012b) Goal-independent mechanisms for free response generation: creative and pseudo-random performance share neural substrates. Neuroimage 59:772-780. CrossRef Medline

Diedrichsen J, Shadmehr R (2005) Detecting and adjusting for artifacts in fMRI time series data. Neuroimage 27:624-634. CrossRef Medline

Dietrich A (2004) The cognitive neuroscience of creativity. Psychonomic Bulletin \& Review 11:1011-1026. CrossRef

Doganaksoy A, Göloglu F (2006) On Lempel-Ziv complexity of sequences. In: Sequences and their applications: SETA 2006 (Gong G, Helleseth T, Song H-Y, Yang K, eds), pp 180-189. Berlin: Springer.

Emery L, Heaven TJ, Paxton JL, Braver TS (2008) Age-related changes in neural activity during performance matched working memory manipulation. Neuroimage 42:1577-1586. CrossRef Medline

Fink A, Benedek M (2013) EEG alpha power and creative ideation. Neurosci Biobehav Rev, in press. CrossRef Medline

Fink A, Graif B, Neubauer AC (2009a) Brain correlates underlying creative thinking: EEG alpha activity in professional vs. novice dancers. Neuroimage 46:854-862. CrossRef Medline

Fink A, Grabner RH, Benedek M, Reishofer G, Hauswirth V, Fally M, Neuper C, Ebner F, Neubauer AC (2009b) The creative brain: investigation of brain activity during creative problem solving by means of EEG and fMRI. Hum Brain Mapp 30:734-748. CrossRef Medline 
Friston KJ, Frith CD, Frackowiak RS, Turner R (1995) Characterizing dynamic brain responses with fMRI: a multivariate approach. Neuroimage 2:166-172. CrossRef Medline

Friston KJ, Buechel C, Fink GR, Morris J, Rolls E, Dolan RJ (1997) Psychophysiological and modulatory interactions in neuroimaging. Neuroimage 6:218-229. CrossRef Medline

Frith CD (2000) The role of dorsolateral prefrontal cortex in the selection of action. In: Control of cognitive processes: attention and performance (Monsell S, Driver J, eds), pp 429-565. Cambridge: MIT.

Halwani GF, Loui P, Rüber T, Schlaug G (2011) Effects of practice and experience on the arcuate fasciculus: comparing singers, instrumentalists, and non-musicians. Front Psychol 2:156. CrossRef Medline

Heuninckx S, Wenderoth N, Swinnen SP (2008) Systems neuroplasticity in the aging brain: recruiting additional neural resources for successful motor performance in elderly persons. J Neurosci 28:91-99. CrossRef Medline

Hyde KL, Lerch J, Norton A, Forgeard M, Winner E, Evans AC, Schlaug G (2009) Musical training shapes structural brain development. J Neurosci 29:3019-3025. CrossRef Medline

Jahanshahi M, Dirnberger G (1999) The left dorsolateral prefrontal cortex and random generation of responses: studies with transcranial magnetic stimulation. Neuropsychologia 37:181-190. CrossRef Medline

Jauk E, Benedek M, Neubauer AC (2012) Tackling creativity at its roots: Evidence for different patterns of EEG alpha activity related to convergent and divergent modes of task processing. Int J Psychophysiol 84:219-225. CrossRef Medline

Kleber B, Veit R, Birbaumer N, Gruzelier J, Lotze M (2010) The brain of opera singers: Experience-dependent changes in functional activation. Cereb Cortex 20:1144-1152. CrossRef Medline

Lau HC, Rogers RD, Ramnani N, Passingham RE (2004) Willed action and attention to the selection of action. Neuroimage 21:1407-1415. CrossRef Medline
Limb CJ, Braun AR (2008) Neural substrates of spontaneous musical performance: an fMRI study of jazz improvisation. PLoS One 3 e1679.

Mayka MA, Corcos DM, Leurgans SE, Vaillancourt DE (2006) Threedimensional locations and boundaries of motor and premotor cortices as defined by functional brain imaging: a meta-analysis. Neuroimage 31: 1453-1474. CrossRef Medline

Nathaniel-James DA, Frith CD (2002) The role of the dorsolateral prefrontal cortex: evidence from the effects of contextual constraint in a sentence completion task. Neuroimage 16:1094-1102. CrossRef Medline

Oldfield RC (1971) The assessment and analysis of handedness: the Edinburgh inventory. Neuropsychologia 9:97-113. CrossRef Medline

Pantev C, Herholz SC (2011) Plasticity of the human auditory cortex related to musical training. Neurosci Biobehav Rev 35:2140-2154. CrossRef Medline

Pantev C, Roberts LE, Schulz M, Engelien A, Ross B (2001) Timbre-specific enhancement of auditory cortical representations in musicians. Neuroreport 12:169-174. CrossRef Medline

Pressing J (1988) Improvisation: methods and models. In: Generative processes in music (Sloboda JA, ed), pp 129-178. New York: OUP.

Salami A, Rieckmann A, Fischer H, Bäckman L (2014) A multivariate analysis of age-related differences in functional networks supporting conflict resolution. Neuroimage 86:150-163. CrossRef Medline

Schneider P, Scherg M, Dosch HG, Specht HJ, Gutschalk A, Rupp A (2002) Morphology of Heschl's gyrus reflects enhanced activation in the auditory cortex of musicians. Nat Neurosci 5:688-694. CrossRef Medline

Smith SM (2002) Fast robust automated brain extraction. Hum Brain Mapp 17:143-155. CrossRef Medline

Steele CJ, Bailey JA, Zatorre RJ, Penhune VB (2013) Early musical training and white-matter plasticity in the corpus callosum: evidence for a sensitive period. J Neurosci 33:1282-1290. CrossRef Medline 